\title{
Failure of the Gross Theory of Beta Decay in Neutron Deficient Nuclei
}

\author{
R.B. Firestone ${ }^{1, e-m a i l: ~ r b f i r e s t o n e @ l b l . g o v ~}$ \\ ${ }^{1}$ Lawrence Berkeley National Laboratory, Berkeley, CA 94720, USA
}

\begin{abstract}
The neutron deficient isotopes ${ }^{117-121} \mathrm{Xe},{ }^{117-124} \mathrm{Cs}$, and ${ }^{122-124} \mathrm{Ba}$ were produced by a beam of ${ }^{28} \mathrm{Si}$ from the LBNL SuperHILAC on a target of ${ }^{n a t}$ Mo. The isotopes were mass separated and their beta decay schemes were measured with a Total Absorption Spectrometer (TAS). The beta strengths derived from these data decreased dramatically to levels above $\approx 1 \mathrm{MeV}$ for the even-even decays; 3-4 MeV for even-Z, odd-N decays; 4-5 MeV for the odd-Z, even-N decays; and 7-8 MeV for the odd-Z, odd-N decays. The decreasing strength to higher excitation energies in the daughters contradicts the predictions of the Gross Theory of Beta Decay. The integrated beta strengths are instead found to be consistent with shell model predictions where the single-particle beta strengths are divided amoung many low-lying levels. The experimental beta strengths determined here have been used calculate the half-lives of 143 neutron deficient nuclei with $\mathrm{Z}=51-64$ to a precision of $20 \%$ with respect to the measured values.
\end{abstract}

\section{Introduction}

Statistical models like Brink-Axel [1, 2], based on the Giant Dipole Resonance (GDR), have proven extremely applicable for the description E1 $\gamma$-ray transitions from excitations where the level density is very high. The success of these models led to the Gross Theory of beta decay [3] assuming that the beta strength should increase with increasing level density and proximity to the Giant Gamow Teller (GT) resonance. However, statistical models have failed to describe M1 $\gamma$-ray transitions, which seem better explained by single-particle models. Beta decay is analogous to M1 $\gamma$-ray decay, connecting primarily levels of the same parity, suggesting that Gross Theory may be an inadequate model. In this paper I will compare measurements of the beta strength function in neutron deficient Xe, $\mathrm{Cs}$, and $\mathrm{Ba}$ isotopes, measured with a Total Absorption Spectrometer, with the predictions of Gross Theory and the shell model.

\section{Experiment}

The isotopes ${ }^{117-121} \mathrm{Xe},{ }^{117-124} \mathrm{Cs}$, and ${ }^{122-124} \mathrm{Ba}$ were produced with a beam of ${ }^{28} \mathrm{Si}$ used to bombard targets of ${ }^{n a t}$ Mo at the LBNL SuperHILAC. These isotopes were isolated the OASIS mass separator system [4], collected on a tape transport system, and deposited inside the LBNL Total Absorption Spectrometer (TAS) [5]. TAS consists of a $36 \times 36 \mathrm{~cm} \mathrm{NaI}(\mathrm{Tl})$ crystal with a $5 \times 20 \mathrm{~cm}$ diameter side well plugged by a $4 \times 15 \mathrm{~cm} \mathrm{NaI}(\mathrm{Tl})$ detector. At the bottom of the well was a $1.6 \times 1 \mathrm{~cm}$ cryogenically cooled germanium $\mathrm{x}$-ray detector. Mass separated sources were transported to the center of the TAS well and counted in $\mathrm{X}-\gamma$ coincidence mode where the EC $\mathrm{x}$-ray energy identified the $\mathrm{Z}$ of the daughter nucleus and the $\gamma$-ray energy sum corresponds to the level energy populated by electron capture decay. Counting intervals were selected on the basis of half-lives and new sources were automatically transported to the counting location at fixed time intervals. The total detection efficiency of TAS for $\gamma$-rays with energies $0.2-10 \mathrm{MeV}$ was $89 \%$ with peak efficiencies ranging from $95 \%$ at $0.3 \mathrm{MeV}$ to $55 \%$ at $5 \mathrm{MeV}$. The lower threshold for sum $\gamma$-ray detection was $200 \mathrm{keV}$. The detector response was modeled using radioactive sources and the EGS4 Monte Carlo code [6].

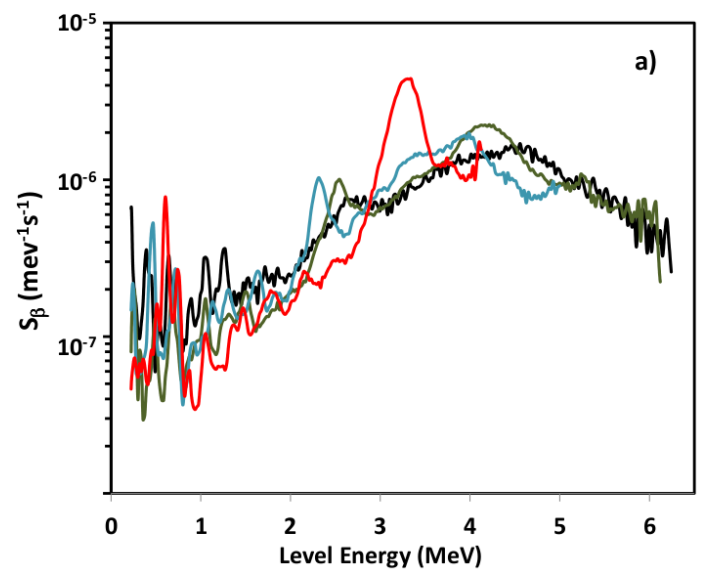

-117 Cs $_{(9 / 2+)}-119$ Cs $_{(9 / 2+)}-121 \mathrm{Cs}(9 / 2+)-123 \mathrm{Cs}_{(1 / 2+)}$

Figure 1: Comparison of beta strengths for the odd-A Cs isotopes. The betas strengths show a significant decline at high energy which is inconsistent with the predictions of Gross Theory.

The decay data for isotopes studied in these analyses are summarized in Table 1. For ${ }^{117,119,121} \mathrm{Cs} \mathrm{J}^{\pi}=9 / 2^{+}$and 
Table 1: Data for the isotopes discussed here. The $\mathrm{J}^{\pi}$ and half-lives are from the Nuclear Wallet Cards [13] and the decay Q-values are from the AME mass evaluation [7, 9]

\begin{tabular}{ccccc}
\hline${ }^{A} \mathbf{Z}$ & $\mathbf{J}^{\pi}$ & $\mathbf{t}_{1 / 2}(\mathbf{s})$ & $\mathbf{Q}_{E C}{ }^{a}$ & {$\left[\sum S_{\beta}(E)\right]^{-1} \equiv \overline{f t}$} \\
\hline${ }^{117} \mathrm{Xe}$ & $5 / 2^{+}$ & 61 & 6251 & 5900 \\
${ }^{117} \mathrm{Cs}$ & $9 / 2^{+}$ & 8.4 & 7690 & 5800 \\
${ }^{118} \mathrm{Xe}$ & $0^{+}$ & 216 & 2892 & 2800 \\
${ }^{118} \mathrm{Cs}$ & $2^{+}$ & 14 & 9670 & 10000 \\
${ }^{119} \mathrm{Xe}$ & $5 / 2^{+}$ & 348 & 4971 & 6200 \\
${ }^{119} \mathrm{Cs}$ & $9 / 2^{+}$ & 43 & 6489 & 5300 \\
${ }^{120} \mathrm{Xe}$ & $0^{+}$ & 2400 & 1581 & 7100 \\
${ }^{120} \mathrm{Cs}$ & $2^{+}$ & 61.3 & 8284 & 9700 \\
${ }^{121} \mathrm{Xe}$ & $5 / 2^{+}$ & 2400 & 3771 & 10800 \\
${ }^{121} \mathrm{Cs}$ & $9 / 2^{+}$ & 122 & 5445 & 7600 \\
${ }^{122} \mathrm{Cs}$ & $1^{+}$ & 21.2 & $7070^{b}$ & 5400 \\
${ }^{122} \mathrm{Cs}$ & $8^{-}$ & 222 & $7210^{b}$ & 9900 \\
${ }^{122} \mathrm{Ba}$ & $0^{+}$ & 117 & 3540 & 3400 \\
${ }^{123} \mathrm{Cs}$ & $1 / 2^{+}$ & 353 & 4205 & 5500 \\
${ }^{123} \mathrm{Ba}$ & $5 / 2^{+}$ & 162 & 5389 & 9100 \\
${ }^{124} \mathrm{Cs}$ & $1^{+}$ & 30.9 & 5930 & 12800 \\
${ }^{124} \mathrm{Ba}$ & $0^{+}$ & 660 & 2642 & 5900 \\
\hline
\end{tabular}

${ }^{a}$ From Wang et al. [7, 9], except as noted, in keV.

${ }^{b}$ From the $\gamma$-ray endpoint, this work.

$\mathrm{J}^{\pi}=3 / 2^{+}$beta-decaying ground state/isomer pairs with similar half-lives exist, but only the $9 / 2^{+}$state dominated the spectrum. Conversely, for ${ }^{118,120} \mathrm{Cs}^{\pi}=2^{+}$and $\mathrm{J}^{\pi}=7^{-}$betadecaying parents exist yet the lower spin state dominated the spectrum. In the case of ${ }^{122} \mathrm{Cs}$ the $1^{+}$and $8^{-}$states GS/isomer states have very different half-lives that could be studied independently by using different counting intervals. In this work the TAS $\gamma$-ray endpoints indicated that the ${ }^{122} \mathrm{Cs}$ decay energy, $\mathrm{Q}_{E C}=7210 \mathrm{keV}$, reported by Wang et al. [7] was found to be for the $\mathrm{J}^{\pi}=8^{-}$isomeric state at an excitation energy of $140 \pm 50 \mathrm{keV}$ so the $\mathrm{J}^{\pi}=1^{+}$state is the GS.

The x-ray coincident TAS $\gamma$-ray spectra corresponds to the relative electron capture feeding to levels in the daughter nucleus. These data can be corrected to the total decay feeding intensity by a factor $\left(f_{E C}+f_{\beta^{+}}\right) / \mathrm{f}_{E C} \mathrm{n}$ [8], and renormalized to $100 \%$ after correction for GS feeding. The experimental beta strength function is then defined as

$$
S_{\beta}(E)=\frac{I_{E C+\beta^{+}}(\%)}{f_{E C+\beta^{+}}(E) t_{1 / 2} \times 100},
$$

where $I_{E C+\beta^{+}}$is binned in $\Delta \mathrm{E}=18.8 \mathrm{keV}$ bins. The odd-A $\mathrm{Cs}$ beta strengths are shown in Fig. 1. Although the beta strength varies widely to low excitation energies, each isotope has comparable beta strength functions to levels at higher excitations. The beta strength were observed to decline rapidly above $\approx 1 \mathrm{MeV}$ for even- $\mathrm{Z}$, even- $\mathrm{N}$ isotopes, 3-4 MeV for the even- $\mathrm{Z}$, odd- $\mathrm{N}$ isotopes. 4-5 MeV for the odd- $\mathrm{Z}$, even- $\mathrm{N}$ isotopes, and 7-8 MeV for the odd$\mathrm{Z}$, odd- $\mathrm{N}$ isotopes. This is inconsistent with the expectation from Gross Theory for a constant $\overline{B(E)}$ where the total beta strength is expected grow rapidly with increasing level density.

\section{Shell model}

The $f t_{1 / 2}$ value is defined as

$$
f t_{1 / 2}=6144 /|M|^{2}
$$

where

$$
|M|^{2}=\left|\int 1\right|^{2}+\left(\frac{C_{A}}{C_{V}}\right)^{2}\left|\int \sigma\right|^{2} .
$$

For mirror transitions with $T=1 / 2$ and $T_{Z}= \pm 1 / 2$ the Fermi matrix element $\left|\int 1\right|^{2}=1$, and for $0^{+} \rightarrow 0^{+}$transitions with $T=1, T_{i z}=1$, and $T_{f z}=0,\left|\int 1\right|^{2}=2$. The ratio of the axial vector and vector coupling constants is $\left|C_{A} / C_{V}\right|=1.24$ from neutron data but approaches unity for heavier nuclei [14]. The Gamow-Teller matrix elements are given by the shell model as [15].

$$
\begin{gathered}
\left|\int \sigma\right|^{2}=\left[\frac{j+1}{j}\right]^{ \pm 1}, j_{i}=j_{f}=l \pm 1 / 2 \\
=4 \frac{l+1 / 2 \pm 1 / 2}{2 l+1}, j_{i}=l \mp 1 / 2, j_{f}=l \pm 1 / 2
\end{gathered}
$$

For the $\mathrm{Xe}, \mathrm{Cs}$, and $\mathrm{Ba}$ decays discussed here both the valence protons and neutrons are in the same $4 \hbar \omega$ oscillator shell. The $0^{+} \rightarrow 0^{+}$transition rate is well determined with $f t_{1 / 2}=3072$ [16], and for mirror transitions $f t_{1 / 2} \approx 4000$ 6000. Although these are well established, simple transitions in light nuclei, it is likely that the same strength should persist in heavier nuclei, especially when the proton and neutron configurations arise from the same shell.

For the experimental decay data an effective $\overline{f t_{1 / 2}}$ can be defined as

$$
\overline{f t_{1 / 2}}=\sum_{E=0}^{Q_{E C}}\left[S_{\beta}(E)\right]^{-1}
$$

and is tabulated in Table 1 . The effective $\overline{f t_{1 / 2}}$ values are all $<13,000$, consistent with $|M|^{2}>0.5$. The decays of even-even ${ }^{118} \mathrm{Xe}$ and ${ }^{122} \mathrm{Ba}$ isotopes have an effective $\overline{f t_{1 / 2}}=2800-3400$, comparable to superallowed $0^{+} \rightarrow 0^{+}$ transitions, and five of the odd- $Z$ decays have $\overline{f t_{1 / 2}}$ values consistent with mirror transitions. These results confirm the hypothesis that the total beta strength is explained by simple shell model transitions whose strength is distributed over many levels.

\section{Half-life calculations}

The beta strengths saturate at low energies suggesting that they can be used as standard strengths to calculate the halflives of nearby nuclei, towards the proton drip line. Although those decay energies are significantly larger, the contribution to the half-life of beta decay to the highest excitation energies appears to be negligible and can be ignored. I have calculated the half-lives for 143 nuclei with $\mathrm{Z}=51-64$ using the beta strengths for ${ }^{117} \mathrm{Xe},{ }^{117} \mathrm{Cs},{ }^{118} \mathrm{Cs}$, and ${ }^{122} \mathrm{Ba}$ for each category of odd-even, odd-odd, and even-even isotopes, respectively. The beta strength to the GS is not included in these input beta strength functions and has been left as a free parameter, determined for each 
group of isotopes with the same atomic number $\mathrm{Z}$, oddeven character, and parent $\mathrm{J}^{\pi}$. A comparison of the fitted half-lives with experimental values, based on this work, is shown in Fig. 2, where it is compared with Gross Theory calculations [17]. The agreement between experiment and theory here is excellent with $\chi^{2} / f=1.0$ assuming a theoretical uncertainty of $20 \%$ and comparable agreement is obtained separately for the odd-even, odd-odd, and eveneven groups. The comparison with Gross Theory is substantially worse with an overall theoretical uncertainty of $49 \%, 73 \%$ for even-A nuclei, and $33 \%$ for odd-A nuclei necessary to obtain a $\chi^{2} / f=1.0$.

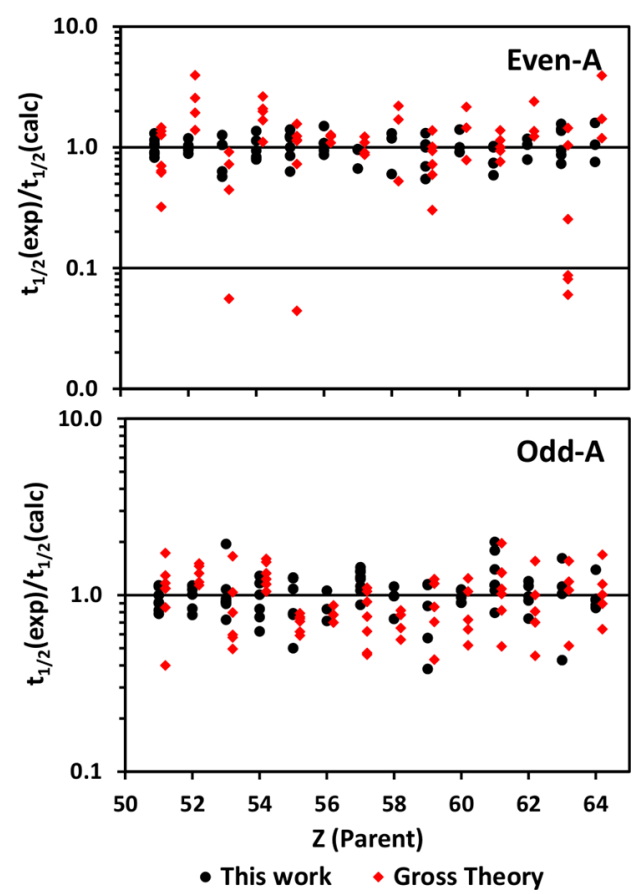

Figure 2: Comparison of experimental and calculated half-lives calculated in this work and with Gross Theory [17] for the neutron deficient isotopes ${ }^{104-117} \mathrm{Sb}$, ${ }^{108-115,117} \mathrm{Te},{ }^{110-117,119,121,123} \mathrm{I},{ }^{111-121} \mathrm{Xe},{ }^{114-125} \mathrm{Cs}$, ${ }^{114-120,122} \mathrm{Ba},{ }^{120-127,129,131,133,135} \mathrm{La},{ }^{121,123-126,128} \mathrm{Ce}$, ${ }^{122-132} \mathrm{Pr},{ }^{127-133} \mathrm{Nd},{ }^{128-137,139,141} \mathrm{Pm},{ }^{129,131-137} \mathrm{Sm}$, ${ }^{134-139,141} \mathrm{Eu}$, and ${ }^{135,137-143} \mathrm{Gd}$.

The experimental beta strengths for the light $\mathrm{Xe}, \mathrm{Cs}$, and $\mathrm{Ba}$ isotopes measured in this work are inconsistent with the predictions from Gross Theory. Little beta strength is observed to high excitations in the daughter nuclei indicating that increasing level density and proximity to the giant GT resonances have little relevance. Instead, these decays can be treated as extensions of the simple shell model transitions observed in light nuclei with comparable beta strengths distributed among many levels below the next shell gap. The beta strengths of the four highest decay energy odd-even, odd-odd, and even-even isotopes were sufficient to predict the half-lives of neutron deficient parents with $\mathrm{Z}=51-64$ to better than $20 \%$ precision.
These results suggest that similar TAS measurements on neutron rich isotopes might also provide beta strength templates for calculating the properties of beta decay in these regions of the nuclear chart. However, those decays typically involve nuclei whose neutrons and protons occupy different oscillator shells where the simple "superallowed" transitions discussed here are blocked. Only the spin flip transitions would be expected to dominate that are observed to be strongly hindered both in light nuclei and near $\mathrm{N}=82$ with $\overline{f t}_{1 / 2}=20,000-50,000$. It remains to be seen whether simple systematics like those found for the neutron deficient isotopes can also be found for neutron rich isotopes.

\section{Acknowledgement}

This manuscript was prepared under Contract No. DEAC02-05CH11231 with the U.S. Department of Energy.

\section{References}

[1] P. Axel, Phys. Rev. 126, 671 (1962)

[2] D. Brink, Ph.D. Thesis, Oxford University (1955)

[3] K. Takahashi and M. Yamada, Progr. Theor. Phys. 41, 1470 (1969)

[4] J.M. Nitschke, Nucl. Instrum Meth. 206, 341 (1983)

[5] M. Karny, J.M. Nitschke, L.F. Archambault, et al., Nucl. Instrum. Meth. Phys. Res. B 126, 411 (1997)

[6] W.R. Nelson, H. Hirayma and D.W.O. Rogers, The EGS4 code system, SLAC Report 265 (1985)

[7] M. Wang, G. Audi, A.H. Wapstra, F.G. Kondev, M. MacCormick, X. Xu and B. Pfeiffer, Chinese Phys. C 36, 1603 (2012)

[8] N.B. Gove and M.J. Martin, Nucl. Data Tables 10, 205 (1977)

[9] G. Audi, F.G. Kondev, M. Wang, B. Pfeiffer, X. Sun, J. Blachot and M. MacCormick, Chinese Phys. C 36, 1157 (2012)

[10] K. Kitao, Y. Tendow and A. Hashizume, Nucl. Data Sheets 96, 241 (2002)

[11] S. Ohya, Nucl. Data Sheets, Nucl. Data Sheets 111, 1619 (2009)

[12] "J. Katakura and K. Kitao, Nucl. Data Sheets 97, 765 (2002)

[13] J.K. Tuli, Nuclear Wallet Cards, National Nuclear Data Center (2011)

[14] O.C. Kistner and B.M. Rustad, Phys. Rev. 114, 1359 (1959)

[15] A. de-Shalit and I. Talmi, Nuclear Shell Theory, Academic Press, New York (1963)

[16] J.C. Hardy and I.S. Towner, arXiv 1312.3587, nuclex (2013)

[17] Nuclear Data Center, Japan Atomic Energy Agency, http://wwwndc.jaea.go.jp/nucldata/index.htm (2013) 
